\title{
Tuning magnetoresistance and electrical resistivity by enhancing localization length in polyaniline and carbon nanotube composites
}

\author{
NARENDRA TANTY* ${ }^{*}$, ANANYA PATRA®, KRISHNA PRASAD MAITY® and V PRASAD \\ Department of Physics, Indian Institute of Science, Bangalore 560 012, India \\ *Author for correspondence (narendra.tanty@gmail.com)
}

MS received 4 October 2018; accepted 3 March 2019

\begin{abstract}
We report low temperature electrical resistivity and magnetoresistance (MR) measurements of conducting polyaniline (PANI) and multiwalled-carbon nanotube (MWCNT) composites. We have used an in-situ oxidative polymerization method to synthesize hydrochloric acid-doped PANI composites with MWCNT weight percentages of 0, 5, 10 and 15. The temperature dependence of resistivity is studied from room temperature to $4.2 \mathrm{~K}$ and analysed by a Mott variable range hopping $(\mathrm{VRH})$ model. The resistivity increases from $1.1 \times 10^{-3} \Omega \mathrm{m}$ at $300 \mathrm{~K}$ to $65.75 \Omega \mathrm{m}$ at $4.2 \mathrm{~K}$, almost four orders of the magnitude change with temperature for pure PANI. Whereas the PANI composite with $15 \%$ MWCNTs shows less variation from $4.6 \times 10^{-4}$ to $3.5 \times 10^{-2} \Omega \mathrm{m}$. The huge change in resistivity is due to the localization of charge carriers in the presence of disorder. At 4.2 K MR shows transition from positive to negative with higher MWCNT loading. Samples with 5 and 10\% MWCNTs show positive MR, whereas the 15\% MWCNT loaded sample shows negative MR. The positive and negative MR are discussed in terms of the wave function shrinkage effect and quantum interference effect on VRH conduction.
\end{abstract}

Keywords. Resistivity; magnetoresistance; disorder; localization.

\section{Introduction}

Organic magnetoresistance (MR) is a hot topic of research in recent years $[1,2]$. From the past few decades organic materials have attracted the attention of spintronics research community due to their low spin-orbit coupling and comparatively a large spin relaxation time as compared to their inorganic counterparts [3-6]. Together with their low-cost production, mechanical flexibility, bio-compatibility, disposability and versatile methods of synthesis, organic materials show a promising potential for spintronics applications [7]. Among organic materials, conducting polymers have been extensively studied for various electronic applications like organic light emmiting diodes [8], gas and chemical sensors [9], supercapacitors [10], batteries [11], organic photovoltaics [12], microwave absorbers [13], transistor applications [14], electromagnetic shielding [15], etc. Their physical properties like electrical and thermal conductivity, thermoelectric power, magnetic susceptibility and optical absorption coefficient have been intensively explored $[16,17]$. However, to make organic spin devices practical with conducting polymers is a challenging task. Researchers need to understand the fundamental concepts of organic MR and charge transport in conducting polymers, which is much more complicated than inorganic materials. There are two different kinds of MR effects that occur in organic devices: (i) organic spin valve effect and (ii) organic magnetoresistance (OMAR) [18]. In the case of organic spin valves, a thin film of the organic material is sandwiched between two ferromagnetic electrodes with different coercivities. From the magnetic electrode, spinpolarized electrons are injected and electric current with a particular spin orientation of electrons flows in the organic material. By applying a suitable magnetic field, the magnetization of electrodes can be changed from the parallel to anti-parallel orientation, which leads to a significant increase in electrical resistivity due to an increase in scattering of opposite spins at the second electrode. Depending on the thickness spin-polarized electrons can either tunnel between the electrodes or can flow diffusively with spin relaxation. In the first case where electrons can tunnel between the electrodes the MR offered is called tunneling MR and in the second case it is named as giant MR. Whereas in the OMAR effect the organic material itself shows a change in resistance on the application of the magnetic field without any magnetic electrodes and spin-polarized electrons. Understanding the OMAR is very much important for spin devices as this phenomenon is not dependent on electrode magnetization and it will have severe impact on the performance of the spin device. Moreover, charge transport properties show less importance when it comes to device fabrication [19]. Conducting polymers are disordered materials with very low carrier mobility and low conductivity which can reduce the device performance significantly. The electrical conductivity of conducting polymers depends on a parameter called localization length which is a measure of disorder in the system [20-22]. However, by adding carbon nanotubes (CNTs) the localization 
length can be enhanced and so as the electrical conductivity. Understanding the effect of disorder on the electrical conductivity and OMAR is crucial for spintronics device application. In this paper we present the OMAR and electrical conductivity studies of polyaniline (PANI) and CNT composites in terms of the localization length.

\section{Experimental}

\subsection{Synthesis of PANI and CNT composites}

PANI-CNT composite samples are synthesized by the method of in situ oxidative polymerization [23]. One millilitre aniline monomer is mixed with $10 \mathrm{ml}$ of $1 \mathrm{M} \mathrm{HCl}$ solution and the required amounts of CNTs are added to the solution. The solution is kept for ultrasonication for $2 \mathrm{~h}$. Another solution of $2 \mathrm{~g}$ of ammonium persulphate and $8 \mathrm{ml}$ of $1 \mathrm{M} \mathrm{HCl}$ is prepared by stirring for $1 \mathrm{~h}$. Aniline solution with CNTs is kept in a bath of the ice and salt mixture to maintain temperature between -4 and $0^{\circ} \mathrm{C}$ and stirred vigorously by a magnetic stirrer. Then ammonium persulphate solution is added to the above solution dropwise. Ammonium persulphate is an oxidizing reagent which initiates the polymerization process. The solution is stirred for $24 \mathrm{~h}$ allowing for the complete polymerization of aniline. Then the precipitated composite powder is collected and washed with methanol and distilled water to remove the residual oligomers. After washing the powder is kept for drying at $60^{\circ} \mathrm{C}$ in a vacuum oven for $48 \mathrm{~h}$ and samples are collected. We have synthesized PANI samples containing $0,5,10$ and $15 \%$ CNTs by this method.

\subsection{Characterization}

2.2a Scanning electron microscopy (SEM): The morphological studies of the samples have been carried out by SEM. Figure 1 shows the SEM image of pure PANI and PANICNT composites with 5, 10 and $15 \%$ CNT loadings. The microstructure of pure PANI is like flakes and globular shaped varying in the size from 10 to $200 \mathrm{~nm}$. From the image of PANI-CNT 5\%, it is clear that PANI starts wrapping up CNTs uniformly, while in situ growth of PANI.

As the CNT concentration is further increased the thickness of PANI coating is reduced but PANI is uniformly distributed among CNTs which can be viewed in the image of PANICNT $10 \%$ and PANI-CNT 15\%. The uniform wrapping of CNTs by PANI has a great impact on its electrical transport properties and MR.

2.2b X-ray diffraction $(X R D): \quad \mathrm{XRD}$ of pure PANI, pure multi-walled carbon nanotube (MWCNT) and MWCNTPANI composites are carried out in purpose of obtaining the structural order of the samples. Figure 2 shows the XRD patterns of all the samples. Pouget et al have already shown the diffraction pattern of pure PANI, [24] which exhibits
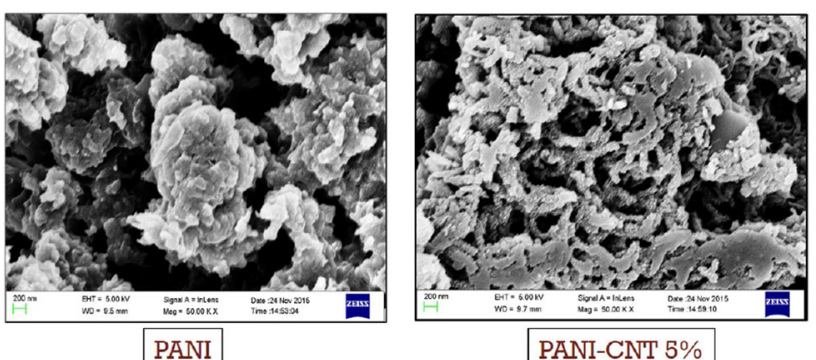

PANI-CNT 5\%
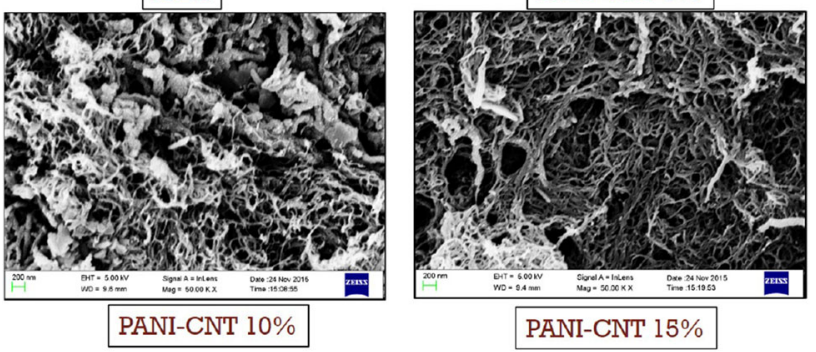

Figure 1. SEM image of PANI-CNT composites.

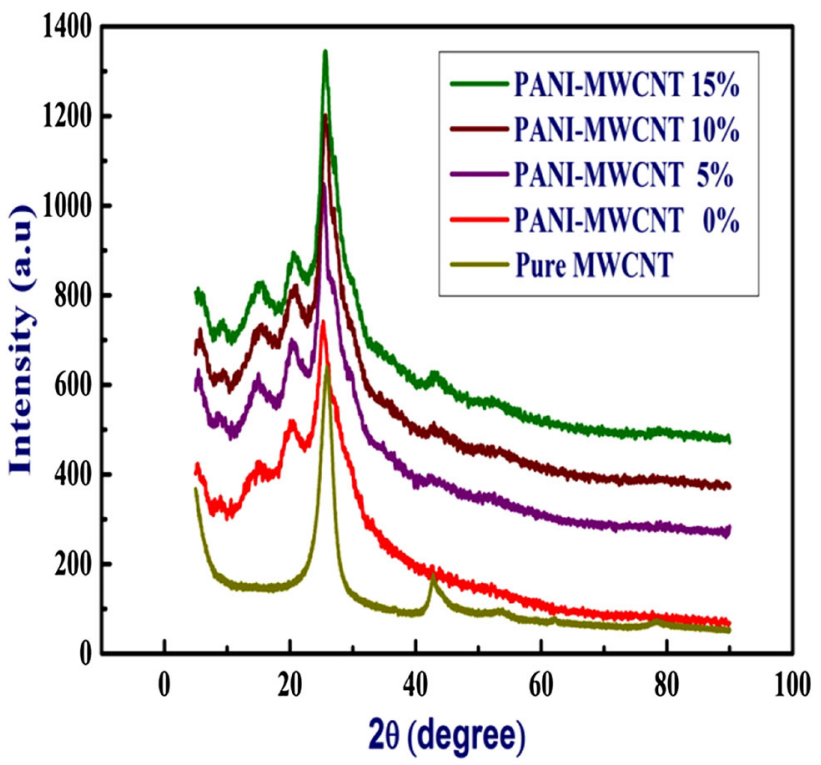

Figure 2. XRD data of CNTs, PANI and PANI-CNT composites with 5, 10 and $15 \%$ loading of CNTs.

peaks at $2 \theta=9.14,14.52,20.2$ and $25.34^{\circ}$. Also the crystalline peak at $25.34^{\circ}$ is more intense than the amorphous peak at $20.2^{\circ}$. And in the case of pure MWCNTs diffraction peaks are observed at $25.94,42.8,53.34$ and $78.4^{\circ}$ associated with (002), (100), (004) and (110) planes of graphite respectively [25]. Comparing the XRD pattern of pure PANI and MWCNT-PANI composites, it is clear that the relative intensity and sharpness of the peaks increase with an increase in MWCNT content. It indicates that an additional crystalline order has been introduced into the composites as aniline polymerizes along the axis of the CNT. 


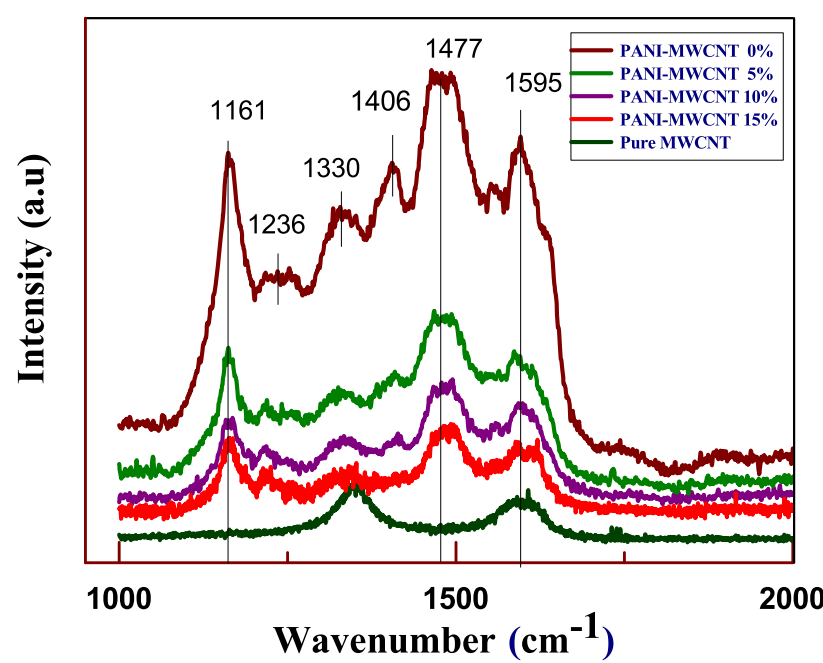

Figure 3. Raman spectroscopy data of CNTs, PANI and PANICNT composites.

2.2c Raman spectroscopy: Raman spectroscopy is one of the most favourite tools to investigate the structure of PANI and also to understand the interaction between PANI and MWCNTs. Figure 3 shows the Raman spectra of PANI-CNT composites. It shows a strong peak at $1161 \mathrm{~cm}^{-1}$ which corresponds to $\mathrm{C}-\mathrm{H}$ in-plane bending in the quinoid ring [26]. The characteristic $\mathrm{C}-\mathrm{N}^{\bullet+}$ stretching vibration of semiquinone radicals at $1331 \mathrm{~cm}^{-1}$ confirms the doping of PANI and hence its conducting form. The $\mathrm{C}-\mathrm{C}$ stretching, $\mathrm{C}=\mathrm{N}$ stretching and $\mathrm{C}=\mathrm{C}$ stretching of the quinoid ring are observed at 1405 , 1478 and $1595 \mathrm{~cm}^{-1}$, respectively. Other small peaks present at 1221 and $1251 \mathrm{~cm}^{-1}$ can be associated with $\mathrm{C}-\mathrm{N}$ stretching in the benzenoid ring and $\mathrm{C}-\mathrm{N}^{\bullet+}$ of semiquinone radicals, respectively.

\section{Results and discussion}

Electrical measurements are performed on the compressed disk-shaped pellets of samples. Pellets with a diameter of $12 \mathrm{~mm}$ are made by applying a pressure of $3 \mathrm{MPa}$. Four-probe direct current resistivity measurement is performed by the van der Pauw method [27], using a Keithley 2400 multimeter and Keithley 850 current source. Low temperature electrical measurements and MR measurements are performed by using a JANIS Cryostat. Measurements are taken up to a liquid helium temperature of $4.2 \mathrm{~K}$ and up to a magnetic field of $5 \mathrm{~T}$ using a superconducting magnet of the cryostat.

\subsection{Resistivity}

Electrical resistivity $(\rho) v s$. temperature $(T)$ measurement is performed for PANI samples with 0, 5, 10 and 15\% CNTs, as shown in figure 4 . The pure PANI sample has the room temperature resistivity of $1.18 \times 10^{-3} \Omega \mathrm{m}$ whereas PANI with $15 \%$

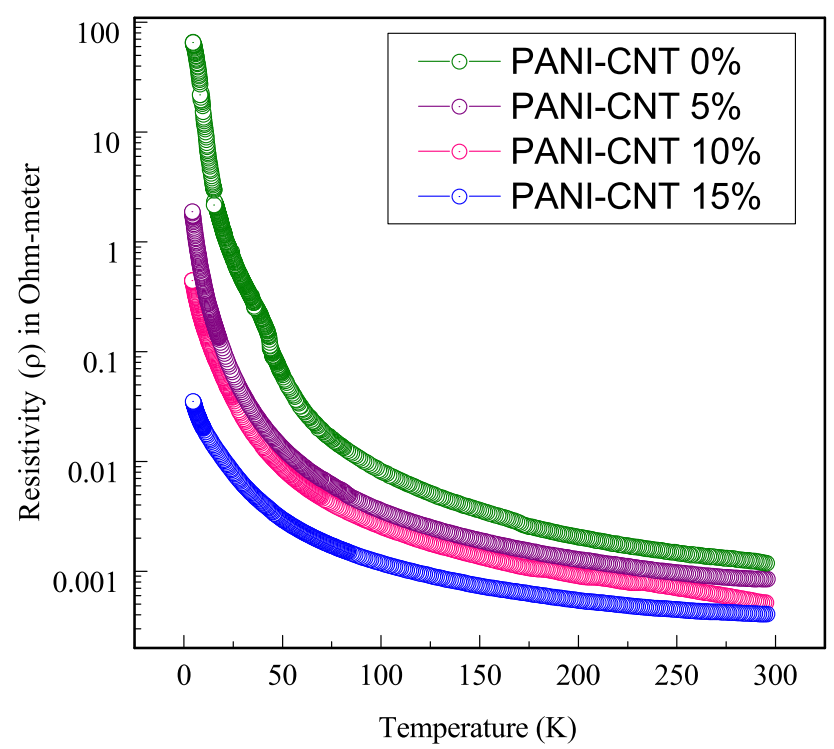

Figure 4. Resistivity vs. temperature graph of PANI and PANICNT composites.

has $4.06 \times 10^{-4} \Omega \mathrm{m}$, one order of magnitude less. The low temperature electrical measurement shows interesting results. All samples show semiconducting behaviour. Resistivity at low temperature increases four orders of magnitude. However, the change in resistivity with temperature is smaller for larger CNT content samples. From the graph it is clear that temperature dependence of resistivity is reduced as the CNT percentage increases in the sample. For pure PANI, resistivity increased from $1.18 \times 10^{-3}$ to $65.575 \Omega \mathrm{m}$ where as for the sample $15 \%$ CNT-PANI, resistivity increased from $4.06 \times 10^{-4}$ to $3.5 \times 10^{-2} \Omega \mathrm{m}$.

The PANI-CNT composite system composed of ordered crystalline region around CNTs and disordered amorphous regions. The crystalline region is metallic in nature and electrical conduction occurs via hopping of charge carriers between the metallic regions. Disorder in the system induces localization of electronic wave function. The wave function decays exponentially after a length $a_{0}$ called as localization length. According to Mott's theory, density of states near band edges will be localized. If the Fermi level lies in the vicinity of localized states then the conduction process has to be of a activated type and the dependence on temperature will be more. This type of conduction can be explained by the Mott variable range hopping (VRH) model [28]. According to this model hopping probability from one localized state to another is

$$
P_{\text {hop }}=v_{\text {ph }} \exp \left(-2 \alpha R_{\text {hop }}-W / \kappa_{\mathrm{B}} T\right),
$$

where $v_{\mathrm{ph}}$ is the phonon frequency, $R_{\text {hop }}$ is the hopping length, $\alpha$ is the inverse of the localization length $a_{0}, \kappa_{\mathrm{B}}$ is 


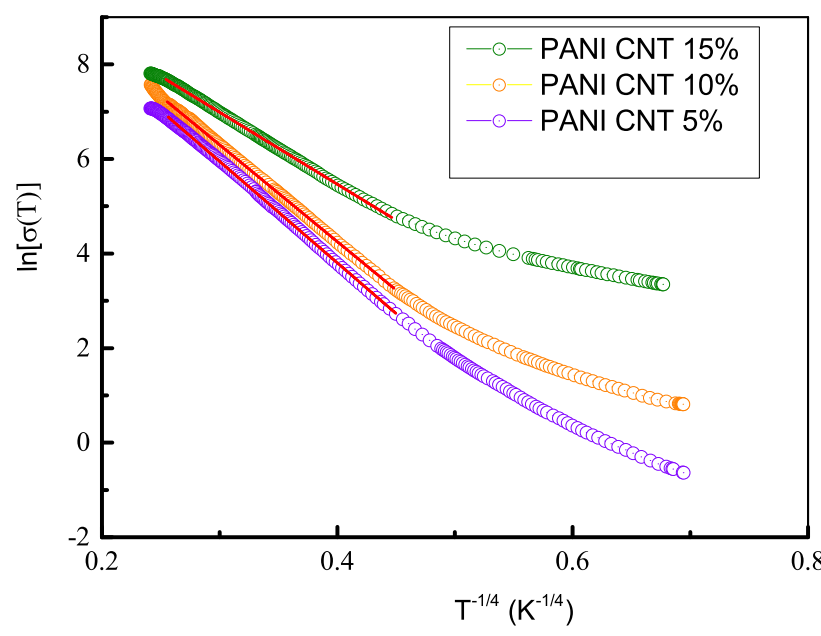

Figure 5. Mott VRH model fitted data of conductivity for PANICNT composites with CNT weight percentages of 5, 10 and 15 .

the Boltzmann constant, $T$ is the temperature and $W$ is the hopping energy:

$$
W=\frac{3}{4 \pi R^{3} N\left(E_{\mathrm{F}}\right)},
$$

where $N\left(E_{\mathrm{F}}\right)$ is the density of states per unit volume per electronvolt at the Fermi energy. In the VRH regime, temperature dependence of conductivity is given by:

$$
\sigma=\sigma_{0} \exp \left[-\left(\frac{T_{0}}{T}\right)^{1 / n+1}\right],
$$

where $\sigma_{0}$ is a constant and represents the conductivity at low temperature limit and $T_{0}$ is the Mott's characteristic temperature which is related to the energy needed for hopping of charge carriers

$$
T_{0}=18 /\left[\kappa_{\mathrm{B}} N\left(E_{\mathrm{F}}\right) a_{0}^{3}\right] .
$$

The characteristic hopping length can be calculated by the formula:

$$
R_{\text {hop }}=(3 / 8)\left(T_{0} / T\right)^{1 / 4} a_{0} .
$$

In order to Motts's law be valid, hopping length $R_{\text {hop }}$ must be greater than localization length $a_{0}$. The Mott's characteristic temperature $T_{0}$ is strongly dependent on the disorder present in the samples. Resistivity vs. temperature data for all samples are fitted by Mott's VRH and corresponding $T_{0}$ is calculated. The VRH fitted curve is shown in figure 5. The localization length can be independently calculated from MR data.

However, below a critical temperature Mott's law is not valid due to the presence of Coloumb interaction between the

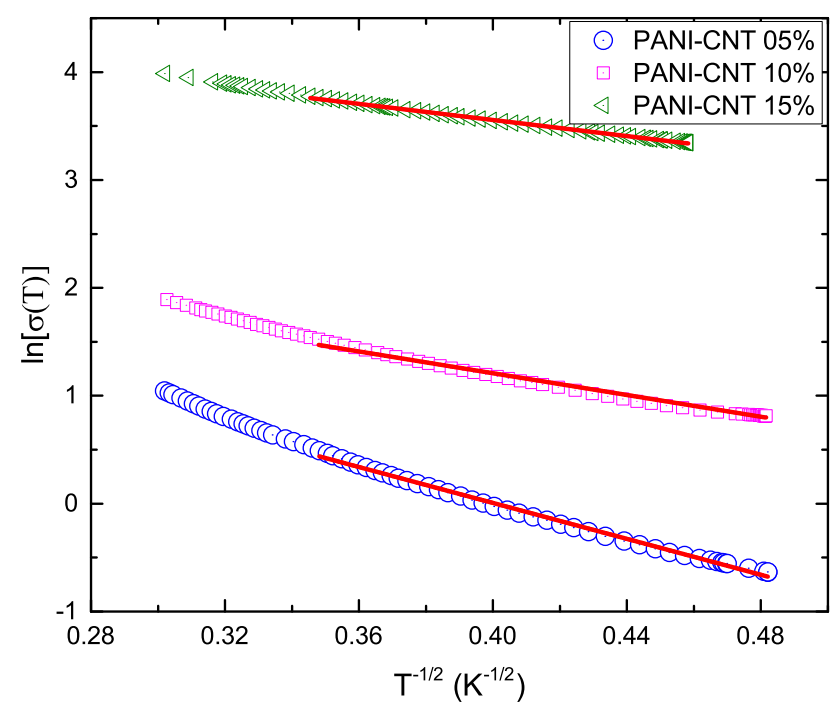

Figure 6. Efros and Shlovskii model fitted data of conductivity of PANI-CNT composites with 5, 10 and $15 \%$ of CNT loading.

localized electrons. At very low temperature the Efros and Shklovskii (ES) model is used to fit the data [29]. In this model the electron-electron interaction is taken into account which introduces a gap at the Fermi level called Coulomb gap $\left(\Delta_{\mathrm{C}}\right)$.

$$
\sigma=\sigma_{0} \exp \left[-\left(\frac{T_{\mathrm{ES}}}{T}\right)^{1 / 2}\right]
$$

where $T_{\mathrm{ES}}$ is the characteristic temperature given by:

$$
T_{\mathrm{ES}}=2.8 e^{2} / \epsilon a_{0} \kappa_{\mathrm{B}} .
$$

The data fitted with the ES model is shown in figure 6. All the parameters like Mott characteristic temperature, Shlovskii characteristic temperature, Coulomb gap and density of states at Fermi energy have been evaluated and presented in table 1 . It is clear from the table that with an increase in CNT content the value of $T_{0}$ decreases from $2.12 \times 10^{5}$ to $5.3 \times 10^{4}$, which indicates that disorder in the sample decreases with CNT content. The localization length is enhanced from 2.78 to $10.84 \mathrm{~nm}$ and hence the resistivity is decreased. Also the value of the Coulomb gap is reduced from 0.1 to $0.01 \mathrm{meV}$. As a result of reduction in the Coulomb gap the value of $T_{\mathrm{ES}}$ is reduced from 69.31 to $13.61 \mathrm{~K}$. All these parameters can be tuned by increasing CNT content and hence the electronic transport can be modified.

\subsection{Magnetoresistance (MR)}

MR measurement is performed for 5, 10 and $15 \%$ CNT loaded PANI samples at a temperature of $4.2 \mathrm{~K}$ up to a magnetic field of $5 \mathrm{~T}$, which is shown in figure 7. MR shows transition 
Table 1. Mott VRH model and ES VRH parameters.

\begin{tabular}{|c|c|c|c|c|c|}
\hline Sample (\%) & $T_{0}$ in $\mathrm{K}$ & $T_{\mathrm{ES}}$ in $\mathrm{K}$ & $\Delta_{\mathrm{C}}$ in $\mathrm{meV}$ & $a_{0}$ in nm & $N\left(E_{\mathrm{F}}\right)$ \\
\hline PANI-CNT 5 & $2.12 \times 10^{5}$ & 69.31 & 0.1 & 2.78 & $4.7 \times 10^{25}$ \\
\hline PANI-CNT 10 & $1.75 \times 10^{5}$ & 25.42 & 0.02 & 3.32 & $3.32 \times 10^{25}$ \\
\hline PANI-CNT 15 & $5.3 \times 10^{4}$ & 13.61 & 0.01 & 10.84 & $3.05 \times 10^{24}$ \\
\hline
\end{tabular}

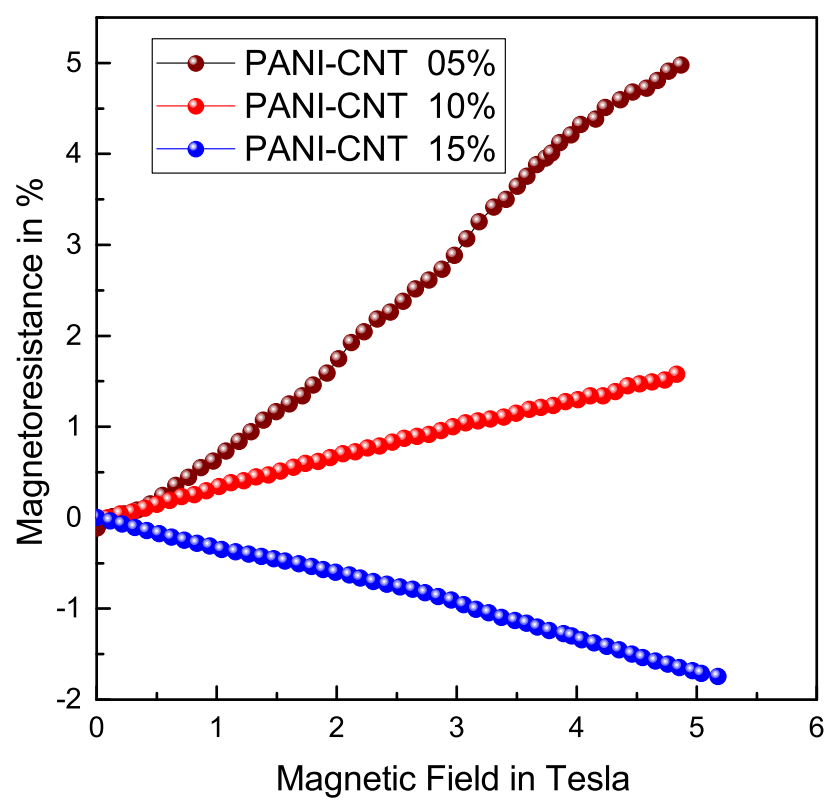

Figure 7. MR of PANI-CNT composites with CNT weight percentages of 5,10 and 15 .

from positive to negative as we increase CNT loading. Lower CNT-loaded PANI samples such as 5 and $10 \%$ samples show positive MR whereas the $15 \%$ CNT PANI sample shows negative MR. Figure 4 shows the graph of MR vs. magnetic field. For 5 and $10 \%$ CNT loaded samples, a change in MR is about 4 and $1.5 \%$ respectively, which is positive. However as CNT loading is increased the MR become negative. For the higher $15 \%$ CNT loaded sample a change in MR is about $1.5 \%$ which is nagative. According to Long et al MR of pure CNTs shows a transition from a negative to positive value [30]. However at a low magnetic field it is negative. Pure PANI shows positive MR and slowly move towards negative MR with an increase in the CNT concentration in the polymer. Positive and negative MR of PANI-CNT samples can be explained by a wave function shrinkage model and forward interference model [31].

3.2a Wave function shrinkage model: By applying a magnetic field, the wave function of localized electrons get squeezed in the transverse direction and overlap between wave functions of the neighbouring site is reduced. So the hopping probability is reduced causing an increase in resistivity and positive MR is observed. The theory of wave function shrinkage was given by Efros and Shklovskii. The expression

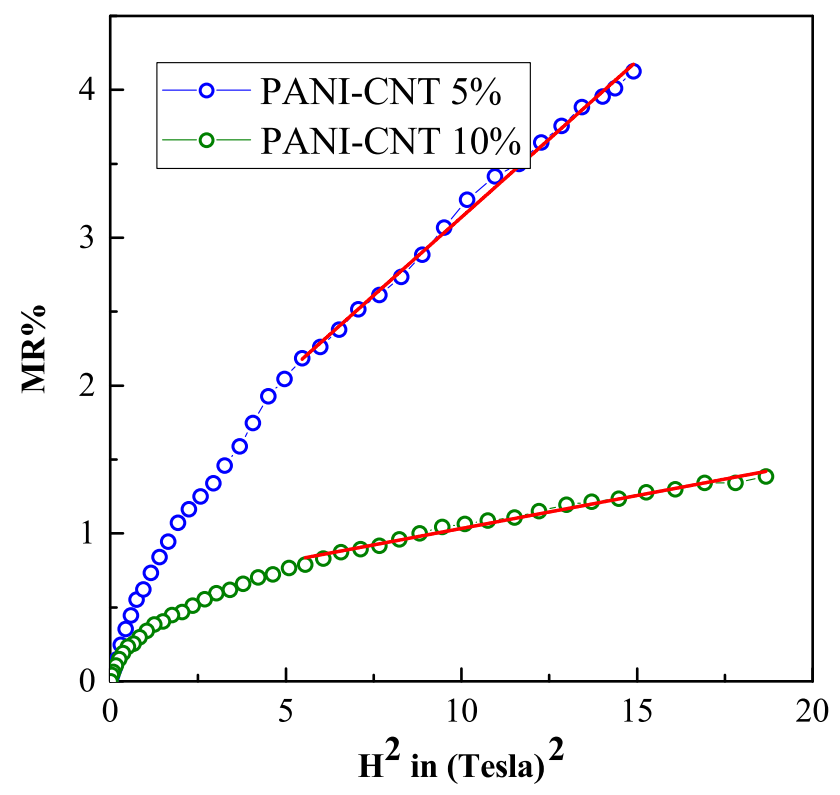

Figure 8. Wave function shrinkage model fitted data for MR of PANI-CNT 5\% and PANI-CNT 10\% samples.

for MR by this model is

$$
\mathrm{MR}=t_{2} \frac{e^{2} a_{0}^{4}}{36 \hbar^{2}}\left(\frac{T_{0}}{T}\right)^{3 / 4} H^{2}
$$

where $t_{2}$ is a numerical constant $\approx 5 / 2016$ and $H$ is the magnetic field. By fitting the MR data with $H^{2}$, the localization length $a_{0}$ can be calculated by fitting the graph as shown in figure 8 . We have calculated the localization length for the 5 and $10 \%$ CNT loaded sample which is found to be 6 and $7.5 \mathrm{~nm}$, respectively.

3.2b Forward interference model: According to the forward interference model, the electron hopping from one site to another experiences various paths and conductivity is proportional to the sum of contributions due to all possible paths. Since there will be an interference effect of various paths, electrons get localized which is called as weak localization. The magnetic field can suppress the destructive interference term leading to reduction in resistivity and hence negative MR. By taking logarithmic average of all possible paths, a linear negative MR is obtained for the lower magnetic field. The forward interference model was first proposed by 


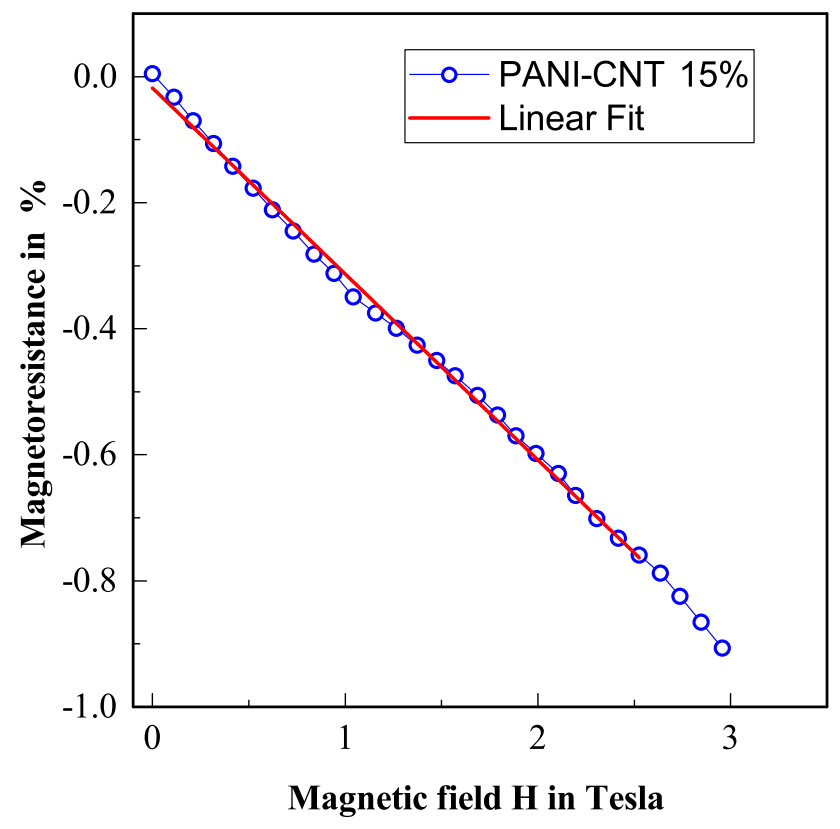

Figure 9. MR data fitted with the forward interference model for the sample PANI-CNT $15 \%$.

Nguyen, Spivak and Shklovskii considering the interference effect of different paths of hopping [31]. The resistance ratio $r_{\text {forward }}=R(H) / R(0)$ is given by the formula:

$$
r_{\text {forward }} \approx 1 /\left\{1+C_{\text {sat }}\left[H / H_{\text {sat }}\right] /\left[1+H / H_{\text {sat }}\right]\right\},
$$

where $R(H)$ is the resistance with the magnetic field $H, R(0)$ is the resistance without the magnetic field and $C_{\text {sat }}$ and $H_{\text {sat }}$ are the fitting parameters. $C_{\text {sat }}$ is the saturation constant and $H_{\text {sat }}$ is the effective saturation magnetic field. For the Mott VRH system $H_{\text {sat }}$ is given by:

$$
H_{\mathrm{sat}} \approx 0.7\left(\frac{8}{3}\right)^{3 / 2}\left(\frac{1}{a_{0}^{2}}\right)\left(\frac{h}{e}\right)\left(\frac{T}{T_{0}}\right)^{3 / 8}
$$

and MR is given by:

$$
\mathrm{MR} \approx-C_{\mathrm{sat}}\left[H / H_{\mathrm{sat}}\right]
$$

By fitting the resistance ratio $r_{\text {forward }}$ according to equation (9) $H_{\text {sat }}$ and $C_{\text {sat }}$ can be calculated. From equation (10) the localization length can be calculated. We need a higher magnetic field to obtain a saturation magnetic field which we can not attain due to the experimental limitation of an instrument. So we could not calculate the localization length from this model. However, we have fitted data, which is shown in figure 9 .

\section{Conclusions}

PANI and CNT composite samples were prepared by an in situ chemical oxidative polymerization method and characterized by XRD and Raman spectroscopy. Low temperature electrical resistivity measurement was performed. Resistivity $v s$. temperature data follow the Mott variable range hopping model. At very low temperature below $15 \mathrm{~K}$ electron-electron interaction comes into picture and it follows the Efros and Shkolvskii model. The higher CNT content sample shows lower $T_{0}$ and enhanced localization length, which is due to an increase in the crystalline order supported by XRD data. Also CNT loading increases the localization length and enhances the conductivity. The Coulomb gap is reduced by adding CNT in PANI and hence $T_{\mathrm{ES}} \mathrm{MR}$ measurement shows a transition from positive to negative MR with an increase in CNT loading, which is due to the quantum interference of various hopping paths as explained by the forward interference model. The localization length was also independently calculated from MR data which also shows enhancement with CNT content, supporting previous calculation from the Mott VRH model. So CNTs enhance conductivity via enhancing the localization length and reducing the Coulomb gap. Also it introduces transition in MR from positive to negative.

\section{Acknowledgements}

The authors gratefully acknowledge the Department of Physics, Centre for Nano Science and Engineering and Advanced Facilities for Microscopy and Microanalysis at I.I.Sc., Bangalore for the support of research facilities.

\section{References}

[1] Gobbi M and Orgiu E 2017 J. Mater. Chem. C 5 5572

[2] Geng R, Daugherty T T, Do K, Luong H M and Nguyen T D 2016 J. Sci. Adv. Mater. Devices 1128

[3] Oksana Ostroverkhova 2019 Handbook of organic materials for electronic and photonic devices (Sawston, Cambridge, UK: Woodhead Publishing Series) ISBN number 9780857092656

[4] Sun D, Ehrenfreund E and Valy Vardeny Z 2014 Chem. Commun. $\mathbf{5 0} 1781$

[5] Alek Dediu V, Hueso L E, Bergenti I and Taliani C 2009 Nature Mater. 8707

[6] Naber W J M, Faez S and Van Der Wiel W G 2007 J. Phys. D: Appl. Phys. 40 R205

[7] Sanvito S 2007 Nature Mater. 6803

[8] Gustafsson G, Cao Y, Treacy G M, Klavetter F, Colaneri N and Heeger A J 1992 Nature 357347

[9] Janata J and Josowicz M 2003 Nature Mater. 219

[10] Snook G A, Kao P and Best A S 2011 J. Power Sources 1961

[11] Novak P, Muller K, Santhanam K S V and Haas O 1997 Chem. Rev. 97207 
[12] Krebs F C 2009 Sol. Energy Mater. Sol. Cells 93 394

[13] Wong P T C, Chambers B, Anderson A P and Wright P V 1992 Electron. Lett. 281651

[14] Garnier F, Hajlaoui R, Yassar A and Srivastava P 1994 Science 2651684

[15] Wang Y and Jing X 2005 Polymers Adv. Tech. 16 344

[16] Skotheim T A 1997 Handbook of conducting polymers (Boca Raton, Florida, USA: CRC Press) ISBN number 978-1-42004358-7

[17] Kaiser A B 2001 Rep. Prog. Phys. 641

[18] Wohlgenannt M 2012 Phys. Status Solidi RRL 6229

[19] Koopmans B, Wagemans W, Bloom F L, Bobbert P A, Kemerink M and Wohlgenannt M 2011 Phil. Trans. R Soc. A 3693602

[20] Kahol P K, Pererab R P, Satheesh Kumar K K, Geetha S and Trivedi D C 2003 Solid State Commun. 125369

[21] Kahol P K, Pererab R P, Satheesh Kumar K K, Geetha S and Trivedi D C 2003 Synth. Metals 139191
[22] Zuppiroli L, Bussac M N, Paschen S, Chauvet O and Forro L 1994 Phys. Rev. B 505196

[23] Yao Q, Chen L, Zhang W, Liufu S and Chen X 2010 ACS Nano 42445

[24] Jdzefowiczt M E and Epstein A J 1991 Macromolecules 24 779

[25] Maniwa Y, Fujiwara R, Kira H, Tou H, Nishibori E, Takata M et al 2001 Phys. Rev. B 64073105

[26] Wu T-M, Lin Y-W and Liao C-S 2005 Carbon 43734

[27] Schroder D K 2006 Semiconductor material and device characterization (Hoboken, New Jersey, USA: John Willey \& Sons) ISBN number 978-0-471-73906-7

[28] Mott N F and Davis E A 2012 Electronic processes in noncrystalline materials (Oxford: OUP)

[29] Long Y, Zhang L, Chen Z, Huang K, Yang Y, Xiao H et al 2005 Phys. Rev. B 71165412

[30] Long Y Z, Yin Z H and Chen Z J 2008 J. Phys. Chem. C 112 11507

[31] Gu H, Guo J, Yan X, Wei H, Zhang X, Liu J et al 2014 Polymer $\mathbf{5 5} 4405$ 